\title{
Clustering in light nuclei and their effects on fusion and pre-equilibrium processes.
}

\author{
Fabiana Gramegna ${ }^{1, \star}$, Magda Cicerchia ${ }^{1,2, \star \star}$, Daniela Fabris ${ }^{3}$, Tommaso Marchi ${ }^{4}$, Marco Cinausero ${ }^{1}$, Meltem \\ Degerlier $^{5}$, Justin Mabiala ${ }^{1}$, Giorgia Mantovani ${ }^{6}$, Luca Morelli ${ }^{7}$, Michela D'Agostino ${ }^{7}$, Mauro Bruno ${ }^{7}$, San- \\ dro Barlini ${ }^{8}$, Maurizio Bini ${ }^{8}$, Gabriele Pasquali ${ }^{8}$, Silvia Piantelli ${ }^{9}$, Giovanni Casini ${ }^{9}$, Giuseppe Pastore ${ }^{8}$, Diego \\ Gruyer $^{10}$, Pietro Ottanelli ${ }^{8}$, Simone Valdré ${ }^{10}$, Nicla Gelli ${ }^{9}$, Alessandro Olmi ${ }^{9}$, Giacomo Poggi ${ }^{8}$, Emanuele Vardaci ${ }^{11}$, \\ Ivano Lombardo ${ }^{11}$, Daniele Dell'Aquila ${ }^{11}$, Silvia Leoni ${ }^{12}$, Natalya Cieplicka-Orynczak ${ }^{13}$, Bogdan Fornal ${ }^{13}$, Daniele \\ Mengoni $^{2,3}$, Gianmaria Collazuol ${ }^{2,3}$, Antonio Caciollii, ${ }^{2,3}$, Maria Colonna ${ }^{14}$, Akira Ono ${ }^{15}$, and Giorgio Baiocco ${ }^{16}$ \\ ${ }^{1}$ Laboratori Nazionali di Legnaro, 35020 Legnaro (Pd), Italy \\ ${ }^{2}$ University of Padova, Dep. of Physics and Astronomy, 35100 Padova, Italy \\ ${ }^{3}$ INFN Padova, 35100 Padova, Italy \\ ${ }^{4} \mathrm{KU}$ Leuven, Dep. of Phys. and Astron. Instituut voor Kern- en Stralingsfysica, 3001 Leuven, Belgium \\ ${ }^{5}$ Science and Art Faculty, Phys. Dep., Nevsehir Haci Bektas Veli Univ., Nevsehir, Turkey \\ ${ }^{6}$ INFN Ferrara and University of Ferrara, Dep. of Chemistry, Ferrara, Italy \\ ${ }^{7}$ INFN Bologna and University of Bologna, Dep. of Physics and Astronomy, Bologna, Italy \\ ${ }^{8}$ INFN Firenze and University of Firenze, Dep. of Physics and Astronomy, Firenze, Italy \\ ${ }^{9}$ INFN Firenze, Firenze, Italy \\ ${ }^{10}$ GANIL, Caen, France \\ ${ }^{11}$ INFN Napoli and University of Napoli, Dep. of Physics and Astronomy, Napoli, Italy \\ ${ }^{12}$ INFN Milano and University of Milano, Dep. of Physics and Astronomy, Milano, Italy \\ ${ }^{13}$ Institute of Nuclear Physics, Polish Academy of Sciences Krakow, Poland \\ ${ }^{14}$ Laboratori Nazionali dl Sud, Catania, Italy \\ ${ }^{15}$ Tohoku University, Sendai, Japan \\ ${ }^{16}$ University of Pavia, Dep. of Physics, Pavia, Italy
}

\begin{abstract}
The study of nuclear cluster states bound by valence neutrons is a field of recent large interest. In particular, it is important to study the pre-formation of $\alpha$-clusters in $\alpha$-conjugate nuclei and the dynamical condensation of clusters during nuclear reactions [1-5]. The NUCL-EX collaboration has recently initiated an experimental campaign of exclusive measurements of fusion-evaporation reactions with light nuclei as interacting partners. In collisions involving light systems, the low expected multiplicity of fragments increases the probability of achieving a quasi-complete reconstruction of the event. In particular the formation and decay modes of an excited ${ }^{24} \mathrm{Mg}$ system have been studied through two different reactions, ${ }^{12} \mathrm{C}(95 \mathrm{MeV})+{ }^{12} \mathrm{C}$ and ${ }^{14} \mathrm{~N}(80.7 \mathrm{MeV})+{ }^{10} \mathrm{~B}$, which have been used to produce fused systems with nearly the same mass and excitation energy ( $\sim 60 \mathrm{MeV})$. In particular, even the de-excitation of the Hoyle state in ${ }^{12} \mathrm{C}$ have been studied, both in peripheral (projectiles de-excitation) and in central collisions (six $\alpha$-particles channel). Moreover, a research campaign studying pre-equilibrium emission of light charged particles and cluster properties of light and medium-mass nuclei has been carried out. For this purpose, a comparative study of the three nuclear systems ${ }^{18} \mathrm{O}+{ }^{28} \mathrm{Si},{ }^{16} \mathrm{O}+{ }^{30} \mathrm{Si}$ and ${ }^{19} \mathrm{~F}+{ }^{27} \mathrm{Al}$ has been recently studied using the GARFIELD+RCo $4 \pi$ setup [6]. The experimental data are compared with the predictions of simulated events generated with the statistical models $\left(\mathrm{GEMINI}^{++}\right.$and $\mathrm{HF} l$ ) and through dynamical models like Stochastic Mean Field (SMF) and Antisymmetrized Molecular Dynamics (AMD) and filtered with a software replica of our apparatus in order to take into account the experimental conditions.
\end{abstract}

\section{Introduction}

Nuclear reactions involving heavy-ions permit the formation of transient nuclear systems, which are excited in the continuum, typically well above the particle and fragment emission threshold. This can be considered quite an

\footnotetext{
^e-mail: fabiana.gramegna@lnl.infn.it

$\star \star$ e-mail: magda.cicerchia@lnl.infn.it
}

important and unique opportunity to probe nuclear systems in extreme conditions of density, temperature and isospin ratio, using experimental techniques. The properties of these excited nuclei, however, cannot be measured directly, since extremely short reaction times in the femtosecond range are involved. The information can, therefore, be accessed only through the decay products of the reaction. However, the experimental analyses of such ex- 
periments, which are performed on an event-by-event basis, are very challenging since the products in the exit channels are a large number of particles and fragments, that cannot be fully detected due to the finite size and resolution of the experimental apparatus and the dead layers. However, on the experimental side, complex detectors based on a $\sim 4 \pi$ geometry, with high granularity and low detection and energy thresholds, have been built: in particular, the GARFIELD-RCo multidetector [6] have been used in the experiments described in the present contribution.

Moreover, in the data analysis, particular care has to be devoted to the observables which have to be considered. When dealing with complex nuclear reactions, in fact, it has been demonstrated that inclusive observables can be typically reproduced by different models, even based on very different physical constraints, which may bring to contradictory interpretations. On the contrary, the comparison to exclusive observables, like the particle-particle and particle-fragment correlations becomes an essential tool to study the excited nuclei decay properties in order to extract information on both the properties of the excited sources, the decay mechanisms, and the occurring competing mechanisms. In this contribution, we will present results which were obtained in different measurement campaigns, performed at the Tandem XTU accelerator of the Legnaro National Laboratory.

\section{Experimental Details}

The experiments have been performed at the Laboratori Nazionali di Legnaro, with respectively the ${ }^{12} \mathrm{C}$ and the ${ }^{14} \mathrm{~N}$ plus the ${ }^{16,18} \mathrm{O}$ and ${ }^{19} \mathrm{~F}$ beams provided by the TANDEM-XTU acceleration system. The GARFIELD plus RCo experimental setup, fully equipped with digital electronics, was used $[6,7]$. The apparatus has 488 detecting cells with a geometrical coverage of the order of $80 \%$ of $4 \pi$. A sketch of the layout is shown in Figure 1. In particular, GARFIELD consists of a couple of drift chambers with gas microstrip read-out for the $\Delta \mathrm{E}$ signals, opportunately coupled to $\mathrm{CsI}(\mathrm{Tl})$ scintillation detectors for the residual energy information: the two drift chambers are filled with $\mathrm{CF}_{4}$ gas at a pressure of 50 mbar and cover the polar angles $\theta=30^{\circ}-150^{\circ}$. They are divided in subsectors, each of which covers $\sim 7^{\circ}$ in azimuthal angle; moreover, in each sector four polar angle regions are defined by four $\mathrm{CsI}(\mathrm{Tl})$ scintillators, each of which covers $\sim 14^{\circ}$ in $\theta$. For both chambers the $\Delta \mathrm{E}-\mathrm{E}$ technique allows charge identification of particles and fragments. Light particles (up to $\mathrm{Z}=3$ ) are identified in both charge and mass through the Fast vs. Slow pulse shape analysis (PSA) technique of the $\mathrm{CsI}(\mathrm{Tl})$ signals. The energies of particles and fragments are determined with an accuracy of the order of few percent with a $0.8 \div 1 \mathrm{AMeV}$ threshold.

The forward polar angles $\left(7^{\circ}-17^{\circ}\right)$ are covered by a three-stage device, the Ring Counter (RCo), consisting of an ionization chamber (IC) (filled with $\mathrm{CF}_{4}$ gas at a pressure of 50 mbar) divided in eight azimuthal sectors, each one followed by an eight strip $300 \mu \mathrm{m}$ thick silicon detector ( $\mathrm{Si})$ and $6 \mathrm{CsI}(\mathrm{Tl})$ scintillators. This allows for an angular resolution of about $1.4^{\circ}$ for the polar angle and $22^{\circ}$ for the azimuthal one. The particle and fragment iden-

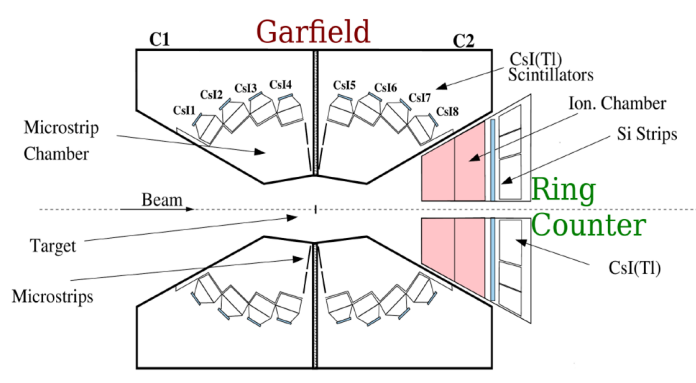

Figure 1: Layout of the GARFIELD + RCo array.

tification is obtained through $\triangle \mathrm{E}-\mathrm{E}$ technique in $\mathrm{IC}-\mathrm{Si}$ or $\mathrm{Si}-\mathrm{CsI}(\mathrm{Tl})$ and/or with CsI(Tl) scintillator fast-slow PSA technique. Using the gas section, particles and fragments are identified in charge with energy thresholds as low as $0.8-1 \mathrm{AMeV}$. Moreover, reaction products characterized by $1 \leq \mathrm{Z} \leq 12$ and energies sufficient to reach the CsI scintillators can be identified in mass with an energy threshold of $\sim 6 \mathrm{AMeV}$. In some cases, mass determination for light fragments stopped in the silicon is also possible, with a quite lower threshold ( 2-3 AMeV), using the PSA technique. A percent accuracy in the determination of the particle and fragment energy is possible in the RCo detector. Due to the high count rate of elastic scattering, the small angles were shielded through an appropriate collimation system. The Evaporation Residues (ER) have been collected in an angular range $\theta=8.6^{\circ}$ and $17^{\circ}$ (just beyond the grazing angles). Coincidences with ER have been selected setting proper gates in the IC vs. Si $\Delta$ E-E energy spectra of the RCo and asking for any coincident particle in the entire apparatus.

\section{The fusion-evaporation case: decay of excited ${ }^{24} \mathrm{Mg}$}

In this paragraph, we will consider the experiments where the following reactions were studied: $a{ }^{12} \mathrm{C}$ beam at 95 $\mathrm{MeV}$ onto a ${ }^{12} \mathrm{C}$ target and a ${ }^{14} \mathrm{~N}$ beam at $80.7 \mathrm{MeV}$ onto a ${ }^{10} \mathrm{~B}$ target. The aim of these measurements consisted in investigating both the reaction mechanisms and the decay of excited nuclei formed in nuclear collisions, by detecting charged particle and studying their correlations and characteristics.

\subsection{Events Selection}

In order to carry out a dedicated analysis, two different event selections have been performed. The first one, related to central events, was fulfilled asking for the detection of the evaporation residue (ER) in the forward direction (RCo) and one or more light particles in coincidence, detected at large $\theta$ angles (GARFIELD). The ER are characterized by a velocity close to the centre of mass velocity of the colliding system [2]. A distribution which correlates the total detected charge vs. the total longitudinal momentum was built and the resulting plot is shown 
in Figure 2 for the ${ }^{12} \mathrm{C}+{ }^{12} \mathrm{C}$ system, where the almost complete fusion-evaporation events are located in the upper right region. Since a large number of events was col-

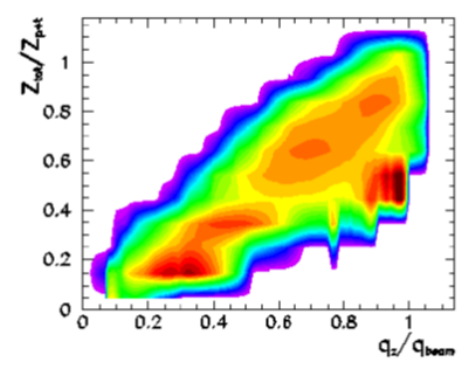

Figure 2: Correlation between the total detected charge and the total detected momentum along the beam axes for the ${ }^{12} \mathrm{C}+{ }^{12} \mathrm{C}$ at $95 \mathrm{MeV}$.

lected, for which the total charge is completely detected, a very strict selection was performed for the subsequent analysis. In particular, only those events were considered, for which both the total incoming charge is $Z_{t o t}=12$ and at least $95 \%$ of the incoming momentum have been measured. It was, however, verified that the less stringent selection $\mathrm{Z}_{t o t} \geq 10$ did not actually bias the characteristics of the events. This second selection was, therefore, used when dealing with specific decay channels, for which more statistics was needed for a complete analysis.

The second event selection was, on the contrary, dedicated to a subset of peripheral events, corresponding to inelastic reactions ${ }^{12} \mathrm{C}+{ }^{12} \mathrm{C} \rightarrow{ }^{12} \mathrm{C}^{\star}+{ }^{12} \mathrm{C}$, which was defined by the detection of three $\alpha$-particles in the forward cone and no charged particles in the remaining solid angle. In this way, the selected channels were related to well defined quantum states of the quasi-projectile and of the quasi-target.

\subsection{Central collisions}

Experimental central events have been compared to Monte Carlo statistical model prediction and, in particular, with the Monte Carlo code HFl, opportunely developed to study the decay of light Compound Systems $[1,8]$. For the ${ }^{12} \mathrm{C}+$ ${ }^{12} \mathrm{C}$ case, a maximum value $\mathrm{J}_{0}^{\max }=18 \hbar$ and a diffuseness parameter $\Delta J=2$ was used for the Angular Momentum in the code. Code prediction are filtered through a software replica of the apparatus, taking into account experimental energy thresholds, dead layers, angular restrictions, energy resolution and solid angle for each detector. The selected sample of experimental data is, in general, compatible with the expected behavior of a complete fusion evaporation reaction. However, some exceptions have been observed, when studying specific decay channels: in particular a larger population have been observed when two or three $\alpha$-particles are emitted in coincidence with an $\mathrm{O}$ or a $\mathrm{C}$ residue, respectively. This is shown in table 1, where, for the ${ }^{12} \mathrm{C}+{ }^{12} \mathrm{C}$ case, for each measured residue, the most probable experimental channel and its branching ratio are compared to the $\mathrm{HF} l$ model predictions. In
Table 1: In the ${ }^{12} \mathrm{C}+{ }^{12} \mathrm{C}$ case, the most probable experimental channel and its branching ratio are compared to the $\mathrm{HFl}$ model predictions for each measured residue. In the case of ${ }^{14} \mathrm{~N}+{ }^{10} \mathrm{~B}$ system, the results for the selected channels with $\mathrm{C}+3 \alpha, \mathrm{O}+2 \alpha, \mathrm{Ne}+\alpha$ respectively are shown for comparison. Errors on the experimental values $(\sim 5 \%)$ are estimated, considering a sum of the statistical errors plus the indetermination due to possible $\alpha$-particle contamination from badly detected ${ }^{3} \mathrm{He}$.

\begin{tabular}{|l|l|l|l|}
\hline & System ${ }^{12} \mathrm{C}+{ }^{12} \mathrm{C}$ & & \\
\hline $\mathrm{Z}_{r e s}$ & Channel & $\mathrm{BR}_{H F l}(\%)$ & $\mathrm{BR}_{E X P}(\%)$ \\
\hline 5 & $11-x n B+x n+p+3 \alpha$ & 100 & 99 \\
6 & $12-x n C+x n+3 \alpha$ & 78 & 98 \\
7 & $15-x n N+x n+p+2 \alpha$ & 95 & 91 \\
8 & $16-x n O+x n+2 \alpha$ & 15 & 63 \\
9 & $19-x n F+x n+p+\alpha$ & 88 & 92 \\
10 & $22-x n N e+x n+2 p$ & 82 & 55 \\
10 & $22-x n N e+x n+\alpha$ & 3 & 26 \\
\hline \hline & System ${ }^{14} \mathrm{~N}+{ }^{10} \mathrm{~B}$ & & \\
\hline $\mathrm{Z}_{r e s}$ & $\mathrm{Channel}$ & $\mathrm{BR}_{H F l}(\%)$ & $\mathrm{BR}_{E X P}(\%)$ \\
\hline 6 & $12-x n C+x n+3 \alpha$ & 77 & 96 \\
8 & $16-x n O+x n+2 \alpha$ & 15 & 56 \\
10 & $22-x n N e+x n+\alpha$ & 3 & 47 \\
\hline \hline
\end{tabular}

particular, both the $\alpha$ energy spectra and the angular distributions in the $(\mathrm{O}+2 \alpha)$ channel are not compatible with statistical model calculations, so that either a contamination from direct reactions or a persistence of $\alpha$-correlations in the ${ }^{24} \mathrm{Mg}$ compound are supposed. However, the total discrepancy with $\mathrm{HF} l$ calculations, cannot be completely ascribed to entrance channel effects, since some discrepancies are also observed when looking at the energy spectrum of $\alpha$-particles in coincidence with $\mathrm{O}$ for the ${ }^{14} \mathrm{~N}$ induced reaction. Even for the $(\mathrm{C}+3 \alpha)$ and the $(\mathrm{Ne}+\alpha)$ channels an anomalously high branching ratio can be observed for both entrance channel systems and they seem to be solely attributed to a possible persistence at high excitation energy of $\alpha$ structure correlations in the ${ }^{12} \mathrm{C}+{ }^{12} \mathrm{C}$ molecular state and/or in the ${ }^{24} \mathrm{Mg}$ compound [2]. This can be observed looking at the results in Table 1 .

\subsection{Hoyle State in ${ }^{12} C^{\star}$}

The data collected in the measurement of ${ }^{12} \mathrm{C}+{ }^{12} \mathrm{C}$ at $95 \mathrm{MeV}$ have been also analyzed in order to investigate the decay of the Hoyle state of ${ }^{12} \mathrm{C}^{\star}$. Two different data selections have been chosen: a first one, which corresponds to peripheral binary collisions, where the quasi-projectile is excited to the Hoyle state and the target has been left in the ground state; a second selection, which is dedicated to the central events, where the formation of a ${ }^{24} \mathrm{Mg}^{\star}$ is considered and the Hoyle state is obtained as a step of the decay chain. A first selection of central events was performed looking for $6 \alpha$-particles events detected by the apparatus, with a complete reconstruction $\left(\mathrm{Z}_{t o t}=12\right)$ of the ${ }^{24} \mathrm{Mg}^{\star}$ decay. To exclude events deriving from different channels than the pure $6 \alpha$-particles, like, for instance, when also a neutron could have been emitted, the dissipated energy was reconstructed through the quantity $\mathrm{Q}_{k i n}$, defined as: $Q_{k i n}=\sum_{i=1}^{6} E_{i}-E_{\text {beam }}$. A selection of events 


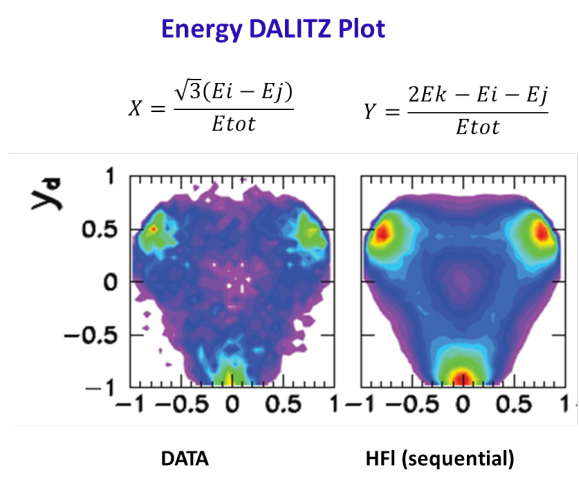

Figure 3: Energy Dalitz Plots: correlations within three $\alpha$-particles. Left panel: Experimental data; Right panel: simulated data from $\mathrm{HFl}$.

have been, therefore, carried out by selecting the opportune $\mathrm{Q}_{\text {kin }}$ region and by analyzing the correlation of three (over six) $\alpha$-particles in each event, to finally choose the minimum excitation energy of the reconstructed ${ }^{12} \mathrm{C}^{\star}$ excitation spectrum (see [9] for details). The characteristics of the Hoyle state decay are very similar in the two samples of data: for example, for the semi-peripheral case, the energy Dalitz plot (see Figure 3) is shown for both the experimental and simulated data, this last derived from the prediction of the statistical evaporation code $\mathrm{HFl}$. Both semi-peripheral and central data sets give similar results and mainly point to a sequential decay through the population of an intermediate ${ }^{8} \mathrm{~B}_{g s}$, with a very small contribution $(\sim 1.1 \%)$ from a simultaneous three $\alpha$-particle process. More details on the particular analysis performed have been presented in the work by L. Morelli et al. [9].

\section{Fusion-Evaporation vs. Pre-equilibrium case: ${ }^{18} \mathrm{O}+{ }^{28} \mathrm{Si},{ }^{16} \mathrm{O}+{ }^{30} \mathrm{Si}$ and ${ }^{19} \mathbf{F}+{ }^{27} \mathrm{Al}$}

In order to continue the extensive experimental program, the comparative study of the four reactions: ${ }^{18} \mathrm{O}+{ }^{28} \mathrm{Si}$, ${ }^{16} \mathrm{O}+{ }^{30} \mathrm{Si}$ and ${ }^{19} \mathrm{~F}+{ }^{27} \mathrm{Al}$ at $7 \mathrm{AMeV}$ and ${ }^{16} \mathrm{O}+{ }^{30} \mathrm{Si}$ at 8 $\mathrm{AMeV}$ has been performed. This is both related to the study of light compound system decay and, furthermore, to the study of pre-equilibrium emission and its possible link to the internal structure of the interacting partners, especially for what it concerns clusters. The main goal of the experiment is to measure and compare the pre-equilibrium component of the particle emission spectra for these systems, especially the $\alpha$-particle emission, since it may be related either to the pre-formation of clusters in nuclei or to their formation during the dynamics of the collision. For central impact parameters, and in the case of complete fusion, the studied reactions all lead to the same compound nucleus, ${ }^{46} \mathrm{Ti}^{\star}$, even if with slightly different excitation energies as shown in Table 2, for which a small difference in the de-excitation chain is expected. On the contrary, choosing the same beam velocity $(7 \mathrm{AMeV})$, the non-equilibrium processes are predicted to be almost the same [10]. From the comparison between statistical and pre-equilibrium light charged particle multiplicities and studying the difference between specific exit
Table 2: Reactions main characteristics.

\begin{tabular}{llllll}
\hline Projectile & Target & $\begin{array}{l}\mathrm{E}_{\text {beam }} \\
(\mathrm{AMeV})\end{array}$ & $\begin{array}{l}\text { Mass } \\
\text { Asymm. }\end{array}$ & $\begin{array}{l}\mathrm{CN} \\
\text { Lab. Vel. }\end{array}$ & $\begin{array}{l}\mathrm{E}^{\star} \\
(\mathrm{MeV})\end{array}$ \\
${ }^{16} \mathrm{O}$ & ${ }^{30} \mathrm{Si}$ & 7 & 0.30 & 1.28 & 88.0 \\
${ }^{18} \mathrm{O}$ & ${ }^{28} \mathrm{Si}$ & 7 & 0.22 & 1.44 & 98.5 \\
${ }^{16} \mathrm{O}$ & ${ }^{30} \mathrm{Si}$ & 8 & 0.30 & 1.37 & 98.4 \\
${ }^{19} \mathrm{~F}$ & ${ }^{27} \mathrm{Al}$ & 7 & 0.17 & 1.52 & 103.5 \\
\hline
\end{tabular}

channels in all the studied systems, even through particleparticle correlations, it will be possible to extract information on the fast emission mechanism and its competition with statistical evaporation from a thermalized system. The influence of possible structure effects, especially those related to the $\alpha$ - clustering in these medium-mass systems, can derive from the comparison of the different entrance channels, where the neutron and proton numbers of the colliding partners have been mutually exchanged. The ${ }^{16} \mathrm{O}+{ }^{30} \mathrm{Si}$ at $8 \mathrm{AMeV}$ have been considered in order to have the same compound nucleus excitation energy of the ${ }^{18} \mathrm{O}+{ }^{28} \mathrm{Si}$ at $7 \mathrm{AMeV}$, hence the same statistical component.

\subsection{Events Selection}

Even in this case, different groups of events have been selected. The first selection, which will be discussed in this paper, is related to central events and it was performed asking for the detection of the ER in the forward direction (RCo) in coincidence with one or more light charged particles in the remaining apparatus. The ER are characterized by a velocity close to the centre of mass velocity of the reaction (see Table 2). A correlation between the detected charge vs. the energy distribution in the Laboratory system for the reaction ${ }^{18} \mathrm{O}+{ }^{28} \mathrm{Si}$ at $7 \mathrm{AMeV}$ is shown in Figure 4 for the total events (left panel) and for the selected central events (right panel) for the experimental data set and for simulated data from SMF+GEMINI [12] and AMD+ GEMINI [13]. See the following paragraph for more details on theoretical calculations. A further selection on a subset of peripheral events is under study, corresponding to inelastic reactions, which can be defined by the detection of four $\alpha$-particles in the forward cone and no charged particles in the remaining solid angle. In this way, we can select channels where the quasi-projectile and the quasi-target are in a well defined single quantum state. This event selection will not be discussed further in the present paper, since the analysis is still too preliminary.

\subsection{Central events: preliminary results}

The energy distributions of the light charged particles detected in coincidence with the residue have been studied and compared to those obtained by model predictions performed either with the statistical model GEMINI ${ }^{++}$ [11], which is based on a complete fusion hyphotesis, or by a dynamical code followed by GEMINI ${ }^{++}$as afterburner. In particular, the comparison to two dynamical 

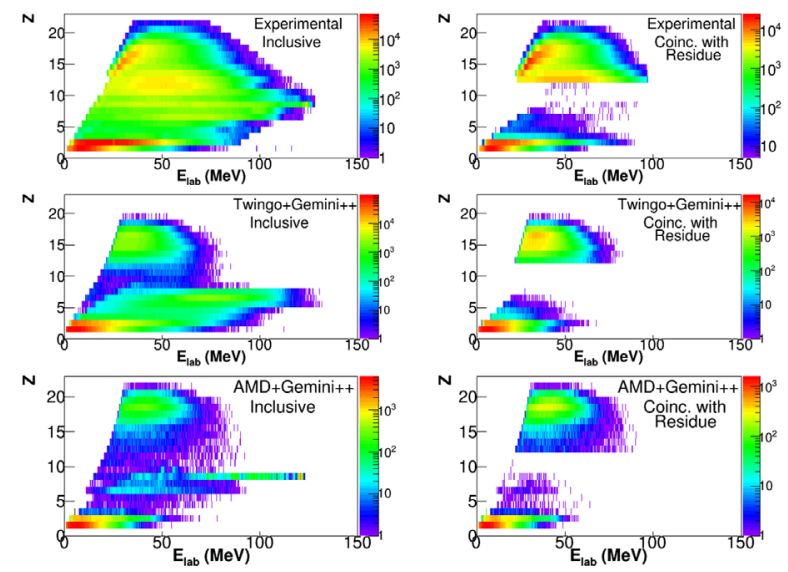

Figure 4: Correlation between the detected charge $\mathrm{Z}$ and the energy $\mathrm{E}$ for total events (left upper panel) and for events where the coincidence between at least one light charged particle in the whole apparatus and one heavy fragment in the RCo (Evaporation Residue) have been selected (right upper panel) for the ${ }^{18} \mathrm{O}+{ }^{28} \mathrm{Si}$ at $7 \mathrm{AMeV}$. The same for the TWINGO+GEMINI (Stochastic Mean Field approach) simulated events in the middle left and right panels. The same for the AMD+GEMINI (Antisymmetric Molecular Dynamics approach) simulated events in the lower left and right panels. Model predictions are filtered through a software replica of the apparatus.

codes are under study: the TWINGO code [12], based on the Stochastic Mean Field approximation, which uses the test particle method to describe each nucleons, and the Antisymmetrized Molecular Dynamics (AMD) [13], which is based on gaussian wave-packets and consider particle-particle correlations with the possibility of describing the cluster structure of the interacting partners. From the preliminary results, one can observe a difference in the four systems when compared to the complete fusion scenario. While, in fact, for $\mathrm{p}, \mathrm{d}, \mathrm{t}$ spectra the complete fusion seems to account for almost all the central collision available cross section, with a minor discrepancy at forward angles expected by the systematics for preequilibrium emission at these low energies, this seems not the case for the $\alpha$-particles. In fact, as it is shown as an example in the Figure 5 for a defined angular region $8.6^{\circ}$ $\leq \theta \leq 13.2^{\circ}$, the results from the GEMINI complete fusion predictions are not in agreement with the data for the $\alpha$-particles. This difference is observed in a large angular range and the gap is growing more and more in the forward detection region (RCo). This result, which could be accounted for by a quite strong and unexpected preequilibrium emission, is essentially related to $\alpha$-particles, since, as already said, a very small amount of fast emission can be attributed to $\mathrm{Z}=1$ particles. Moreover, from the comparison of the different systems with their own model predictions, one can infer that the amount of fast emission seems to be dependent on the system/entrance channel, as one can observe, for example, looking at the ${ }^{19} \mathrm{~F}+{ }^{27} \mathrm{Al}$ at 7 $\mathrm{AMeV}$ case, where the difference between data and GEMINI are relatively smaller.

For what it concerns the dynamical models and, in particular the TWINGO code, unfortunately, some limitations in describing the pre-equilibrium phenomenon are present
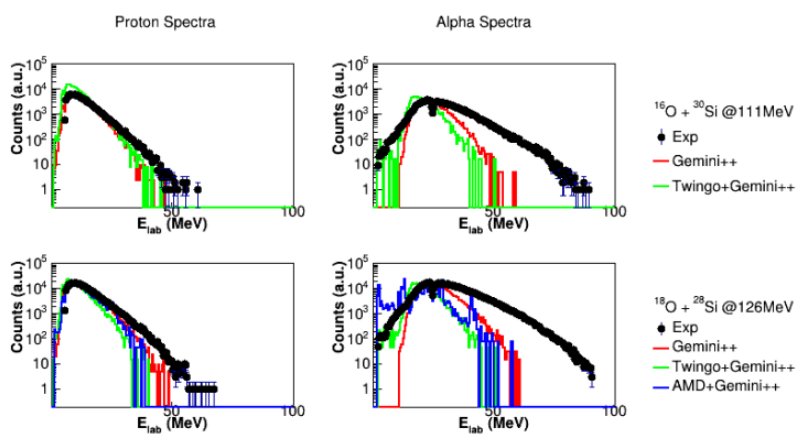
-Twingo+Geminit+ $E_{\text {lab }}(\mathrm{MeV}$

$E_{\text {abo }}(\mathrm{MeV})$
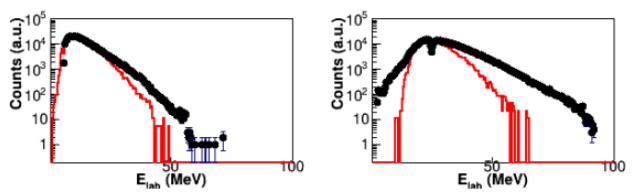

${ }^{16} \mathrm{O}+{ }^{30} \mathrm{Si} @ 128 \mathrm{MeV}$

- Exp
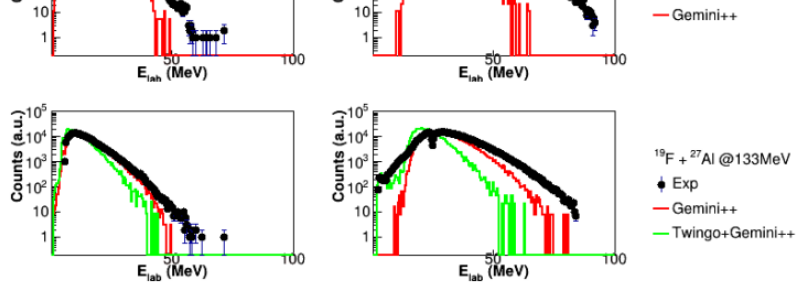

Figure 5: Experimental energy distributions (black full dots) collected at $8.6^{\circ} \leq \theta \leq 13.2^{\circ}$ for protons (left panels) and $\alpha$-particles (right panels), for the four studied reactions, respectively: ${ }^{16} \mathrm{O}+{ }^{30} \mathrm{Si}$ at $7 \mathrm{AMeV}$ (upper panels); ${ }^{18} \mathrm{O}+{ }^{28} \mathrm{Si}$ at $7 \mathrm{AMeV}$ (second upper panels); ${ }^{16} \mathrm{O}+{ }^{30} \mathrm{Si}$ at 8 $\mathrm{AMeV}$ (third upper panels); ${ }^{19} \mathrm{~F}+{ }^{27} \mathrm{Al}$ at $7 \mathrm{AMeV}$ (bottom panels). In all panels comparison to model predictions are provided: GEMINI statistical model (red line) and TWINGO + GEMINI dynamical plus statistical model (green lines). For the case ${ }^{16} \mathrm{O}+{ }^{30} \mathrm{Si}$ at $8 \mathrm{AMeV}$ no TWINGO + GEMINI simulation have still been run, while for the ${ }^{18} \mathrm{O}+{ }^{28} \mathrm{Si}$ at 7 $\mathrm{AMeV}$ case even few events simulated with AMD+GEMINI (blue lines) are compared to experimental data. Model prediction are filtered through a software replica of the apparatus.

since, due to the particle-test method, all nucleons emitted before the equilibration cannot be properly reconstructed in the final event. Moreover, being TWINGO based on Stochastic Mean Field approximation, it is not even able to build up clusters like $\alpha$-particles and, therefore, also these fast particles are missing in the final spectrum. Therefore, only the nucleons and $\alpha$-particles recontructed by the after-burner GEMINI are present in the final distributions: in other words, only the statistical decay of the excited systems produced at $300 \mathrm{fm} / \mathrm{c}$ (selected collision time in our case at which the after-burner is applied) can be accounted for. Since the system has anyway lost some nucleons and clusters in the early stage of the reaction, the TWINGO+GEMINI case results in a colder system population with respect to GEMINI alone: predictions on the fast emission can then be obtained only as a difference to the experimental spectra. The AMD+GEMINI simulation can give different information, as we discussed previously, being able to describe nucleon -nucleon correlations. For the case ${ }^{18} \mathrm{O}+{ }^{28} \mathrm{Si}$ at $7 \mathrm{AMeV}$ we have at present few events simulated with AMD+GEMINI (blue lines in Figure 5, which have been compared to experimental data. However, the statistics is still too low and subject to statistical fluctuations to permit to draw any conclusion at present. Another observable is the Light Charged Particles multiplicity distribution, which are shown in Figure 6 for protons (upper panels) and for $\alpha$-particles (lower 
panels). The multiplicity distributions for both protons and $\alpha$-particles for all systems are different from simulations. In general, for protons GEMINI seems to overestimate the experimental results for all systems, especially in specific channels $(2 \mathrm{p}, 3 \mathrm{p}, 4 \mathrm{p})$. TWINGO predictions are closer to the data for the ${ }^{19} \mathrm{~F}+{ }^{27} \mathrm{Al}$ at $7 \mathrm{AMeV}$ system. For $\alpha$-particles the situation is even more complex and the situation seems to evolve in a very different way from one system to another. This, anyway, is an indication that further and more detailed studies have to be performed on the specific decay channels, to really understand the underlying phenomena, in a similar way like it was performed in the previous experiment. It is, therefore, necessary to use
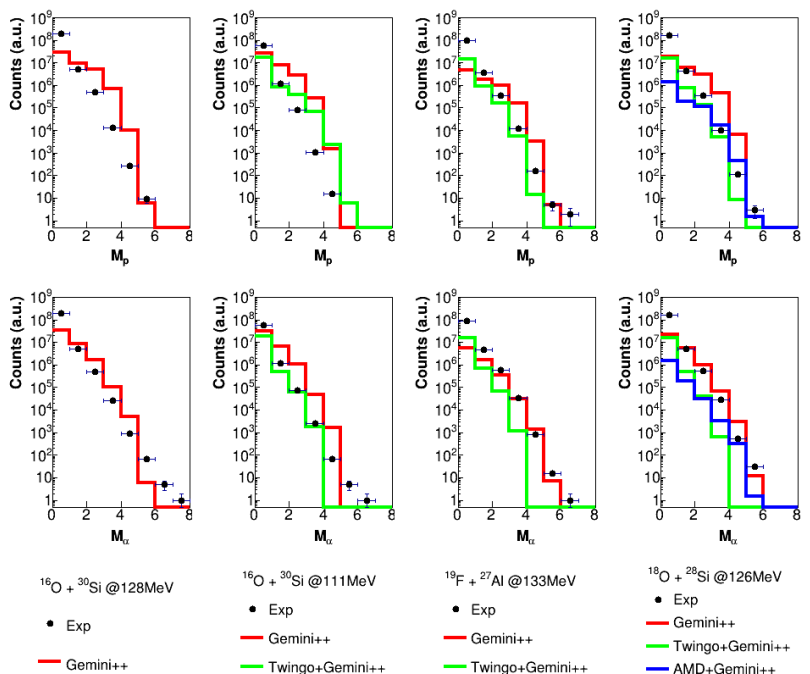

Figure 6: Experimental Light Charged Particle multiplicities (black full dots) in coincidence with ER: proton multiplicity (upper panels) and $\alpha$-particle multiplicity (bottom panels), for the four studied reactions, respectively: ${ }^{16} \mathrm{O}+{ }^{30} \mathrm{Si}$ at $8 \mathrm{AMeV}$ (left panels); ${ }^{16} \mathrm{O}+{ }^{30} \mathrm{Si}$ at $7 \mathrm{AMeV}$ (second left panels); ${ }^{19} \mathrm{~F}+{ }^{27} \mathrm{Al}$ at $7 \mathrm{AMeV}$ (second right panels); ${ }^{18} \mathrm{O}+{ }^{30} \mathrm{Si}$ at $7 \mathrm{AMeV}$ (right panels). In all panels comparison to model predictions are provided: GEMINI statistical model (red line) and TWINGO + GEMINI dynamical plus statistical model (blue lines). Model predictions are filtered through a software replica of the apparatus.

other theoretical models to account for this. On one side, calculations for the different systems are undergoing with the Moscow Pre-equilibrium Model (MPM) [5, 14, 15], which was already used to compare its predictions with previous measured data [3]. This code is based on the Hybrid Exciton Model coupled to a Statistical Decay Code (PACE2). Moreover, the Antisymmetric Molecular Dynamics (AMD) calculations are under study, since they can properly take into account the possible $\alpha$-cluster structure in the colliding systems. AMD is based on a Stochastic Equation of Motion for the Gaussian Wave Packets representing the colliding nucleons: unfortunately the time needed to run the code for a sufficient number of events to compare to exclusive data is long and up to now only global observable have been obtained like the one shown in Figure 4. More time is needed to calculate all the studied systems and to compare the simulated events to exclusive observables in the experimental data. Several calcu- lations may also be needed to change and check the optimized input parameters related to the clusterization factor.

\section{Conclusions}

We have presented complete results for the ${ }^{24} \mathrm{Mg}$ compound system, formed through two different entrance channels. In the case of this light system, the complete charge detection allows a precise identification of the emission source as ${ }^{24} \mathrm{Mg}$, at an excitation energy $\mathrm{E}^{\star}=60$ $\mathrm{MeV}$. We especially focussed on multiple $\alpha$-particle emission channels, since they are believed to bear information on exotic $\alpha$-cluster states, which could be populated at high excitation energy. For the exotic decay channel of a highly excited ${ }^{24} \mathrm{Mg}$ into six $\alpha$-particles, the complex analysis performed, even through correlation function technique, shows that the decay is essentially sequential, due to an important contribution of intermediate Be states. For slightly heavier systems and higher involved energies, the ${ }^{16} \mathrm{O}+{ }^{30} \mathrm{Si}$ at $7 \mathrm{AMeV},{ }^{18} \mathrm{O}+{ }^{28} \mathrm{Si}$ at $7 \mathrm{AMeV},{ }^{16} \mathrm{O}+{ }^{30} \mathrm{Si}$ at $8 \mathrm{AMeV}$ and ${ }^{19} \mathrm{~F}+{ }^{27} \mathrm{Al}$ at $7 \mathrm{AMeV}$ have been studied. The analysis is still very preliminary, even though particular results seem to come out looking at the $\alpha$-decay channels, where already in a quasi-inclusive mode (ER coincidence - no multiple channel selection) unexpected differences are evidenced between the different studied systems for what it concerns the competition between evaporative and fast emission channels. Comparison to specific simulation models like the AMD are under study, to evidence the possible influence of $\alpha$-structure effects in the dynamics of the reactions. Study of more exclusive decay channels, together with pre-equilibrium predictions will be performed in order to get a better insight on the reaction interplay.

\section{References}

[1] L. Morelli et al., Journ. of Phys. G 41, 075107 (2014)

[2] L. Morelli et al., Journ. of Phys. G 41, 075108 (2014)

[3] D. Fabris et al., PoS(X LANSPA , 061 (2013)

[4] V.L. Kravchuk, et al. EPJ WoCs, 210006 (2010)

[5] O. V. Fotina et al., Int. Journ. Mod. Phys. E 191134 (2010)

[6] M. Bruno, F. Gramegna et al., Eur. Phys. Journ. A 49 128 (2013)

[7] Gramegna F. et al., Nucl. Instr. And Meth. A, 389 (1997) 474; Gramegna F. et al., 2004 IEEE Nucl. Science Symposium, Rome, 16-22 October 2004

[8] Baiocco G., PhD Thesis (2012) University of Bologna and University of Caen, Basse-Normandie

[9] L. Morelli et al., J. Phys. G: Nucl. Part. Phys. 43 (2016) 045110

[10] P.E. Hodgson, E. Běták, Phys. Rep. 374 (2003) 1-89.

[11] R. J. Charity, Phys. Rev. C82 (2010) 014610.

[12] M. Colonna et al., Nucl. Phys. A 642, 449 (1998).

[13] A. Ono, Phys. Rev. C59 (1999) 853.

[14] D.O. Eremenko et al. Phys Atom. Nucl. 65 (2002) 18

[15] O.V. Fotina et al. Phys. Atom. Nucl. 73 (2010) 1317c 